\title{
AN INTELLECT LEARNING ON E-MAIL SECURITY AND FRAUD, SPAM AND PHISHING
}

\author{
Dr.P.S.Jagadeesh Kumar ${ }^{1}$, Dr.S.Meenakshi Sundaram², Mr.Ranjeet kumar ${ }^{3}$ \\ 1,2,3 Department of Computer Science and Engineering, \\ Don Bosco Institute of Technology, Kumbalagodu, Bangalore, India - 560074.
}

\begin{abstract}
Cybercrime has grown voluminous pleats with veneration to the development of first-hand technology. The flout towards cybercrime has become todays prime centric with developing countries frugality as well. Nonetheless hefty figure of security and privacy available with modern expertise; phishing, spam and email fraud are more equally exasperating. In this intellect learning, the authors' primary interest is to make a healthy charge on phishing, spam and email fraud towards the wealthy personal information and realm. Official and business related information needs added exhaustive sanctuary and discretion from the hackers to be on the top in their one-to-one arena.
\end{abstract}

\section{KEYWORDS}

Cybercrime, Phishing, Spam, Email fraud, Security and Privacy, Intellect learning.

\section{INTRODUCTION}

Phishing is one of the diverse sorts of fraud un swerving these days. In illicit law, fraud is defined as a thoughtful con made for the solitary objective of delicate advantages or for coating a personage's doppelganger. In wide-ranging footings, fraud can be demarcated as a deed of misleading public into skimpy their private evidence, essentially for the perseverance of monetary or subjective achievements. Phishing bring up to the deed that the in vaderglamor operators to call a counterfeited website by sending them forged e-mails, and surreptitiously get quarry's delicate data such as user label, watchword, and domestic safe keeping credentials, etc. These statistics then can be jumble-sale for imminent bull commercials or even individuality burglary out breaks. Phishing has turn out to be supplementary, byzantine and erudite so that phishers can circumvent the sifter fixed by contemporary hostile phishing practices and troupe their lure to patrons and officialdoms. A conceivable elucidation is to craft a stout classifier to augment the phishing email concealment and look after clienteles from feat such forwards. The doing of directing an e-mail to a consumer deceitfullys uing to be a time-honoured validget-up-and-go in astab to dodge the consumer into yielding own facts that will be castoff for distinctivenesss hoplifting. The e-mail show the way the user to holiday a website where they are probed to refurbish own figures, such as watchwords and credit card, social security, and bank account numbers, that the sincere business by this time partakes. The website is phony and traditional out of bedmerely to snip the consumer's gen.

The impetus of junk mail habitually encompasses returnscohort, sophisticated quest standing, upholding merchandises and amenities, pilferinggen and phishing. Spam may well upshot in detrimental way on the frugality. Some exploration has sued spam interpreted for just about 20 billion dollars in gonestint and throughput. Notwithstanding the exertion vexing to break spam,

DOI : 10.5121/ijnsa.2015.7503 
uncalled-for viable emails never appear to sojourn inward in our email inboxes. Sundry email benefactors, such as Gmail or Hotmail, have meticulously urbanised their spam sieves to spot probable spam emails. Nonetheless, if the sieve fallaciously as certains an imperative missive as spam, it could fashion a delinquent added than impartial an exasperation as consumers' valour blunder an central date or fail to keep an eye on communiqué of prodigious moment. Farther, just about $80 \%$ of the oomph expended was allied to consumers scrubbing spam and incisive for deceitful positives. Spam sieving interpreted for $16 \%$ of such oomph routine, then again efficacious sieving was competent to condense the oomphroutineearnt by spam or else.

\section{LITERATURE ANALYSIS}

Andronicus A.Akinyelu et al [1] clinched that phishing has become a sombre threat to total sanctuary and frugality. The debauched level of advent of first-hand phishing websites and disseminated phishing bouts has made it tough to retain precludes out of bed to epoch. Auxiliary they unfilled a gratified based phishing detection slant which has linked the contemporary fissure branded in the prose. Their tactic conceded high classification correctness of $99.7 \%$ with trifling deceitful optimistic proportion of nearby $0.06 \%$. In the impending, they strategic on taming their toil by conjoining this slant with a nature inspired (NI) technique. NI modus operandi can be rummage-sale to robotically and apathetically ascertain the paramount phishing topographies that can be jumble-sale to physique a stout phishing email sieve with very tall taxonomy exactitude. By means of this modus operandi will with no qualmboost the foretelling exactitude of a classifier since operational cataloguing of emails hinge on the phishing topographies branded during the erudition leg of the taxonomy. Owing to the hasty revolution in phishing attack outlines, up-todate phishing concealment skills prerequisite to be momentously enriched to meritoriously warfare embryonic phishing attacks.

Dhanalakshmi Ranganayakulu et al [2] unfilled that hackers evade anti-spam sieving practices by entrenching malevolent URL in the content of the memoranda. The URL analyser method with the aid of abated phishing feature customary as certains the malevolent URL in the emails. The datasets are gained from two cradles viz DMOZ Open Directory Project and Phishtank (2012). Phishtank is a spring of outlawed phishing URLs which confesses consumer feedbacks and they are also corroborated by consumers. An E-Mail server has been shaped with hMail named as SSE Mail Server for the taxing tenacities. The false positive rate refers to the figure of authentic emails hush-hush as phishing emails, and false negative rate refers to the figure of phishing emails classified as authentic.

Jagruti Patel, Sheetal Mehta et al [3] fathomed that phishing emails have become communaltricky in topicaleons. Phishing is a nature of bout in which fatalities sent emails into which consumers have to afford subtlegen and then unswervingly propelled to the phisher. There are sundry modus operandi for perceiving phishing email but there are precincts of lowaccuracy, content can be same as authentic email so cannot be spotted, revealing rate is not high. So aboutspread method is obligatory. To flabbergast these limitation, amalgam feature medley can be smeared. The skins are centred header gen and URL. By consuming header information, sender's behaviour can be scrutinized. Smearing this slant in imminent, the exactitude and revealingspeed can be dignified.

M.Madhuri, K.Yeseswini et al [4] borne that phishing has made ecommerce doubted and minusstriking to regulartrades. They devouredwilfulphysiognomies of the hyperlinks that were entrenched in phishinge-mails. They formerlypremeditated an anti-phishing algorithm, LinkGuard, based on the imitative physiognomies. Phishing-Guard is distinctive based, it can solitary distinguishnotorious out breaks, but also is operative to the anonymous. Theyinstigated Link Guard for Windows XP. Thetry-outbared that Link Guard is nimble-partisan and canisterdiscoverunto $96 \%$ anonymous phishing outbreaks in here and now. LinkGuard is not merelyexpedient for noticing phishing bouts, but also can armourconsumers from malevolent or 
uncalled-for links in web pages and on the spot messages. Theimminentexertionembraces further outspreading the LinkGuard algorithm, so that it can grip CSS (cross site scripting) attacks.

Tzipora Halevi et al [5] clinched that research scans the dynamics that may subsidise to proneness to wired sanctuary and secrecy bouts. Their revision regarded at the connexion amid persona traits and phishing email rejoinder. It advance studies the connexion amongst online behaviour and the likelihood of being phished. The outcomes have imperative insinuations, as they spectacle that definite persona traits may rootadvanced phishing openness. Precisely, this learning stablish that womenfolk may be more inclined to flagship phishing bouts than kinsmen. This recommends phishing ramparts should be couturiernear society who groove high on firm persona traits. This exertion also catches that publics who are more betrothed with Facebook commotion also have less deterring privacy settings and consequently may be extradefenceless to conceal mentcoercions. This submits people who centre more on the doles of Facebook incline to snub its jeopardies, a cause that should be painstaking when bidding to nurture cognizance about privacy seepages through user edification. Future work should quintessence on email phishing attacks with diverse email categories. The email was a flagship email, therefore alluringto appetite. The expressiveimpetuses for retorting to unalike email types may be poles apart. Leeway for impending work is to auto-recommend apposite privacy settings to the consumers grounded on their persona backgrounds.

Jayshree Hajgude et al [6] urbanised anamalgamroutine to perceive phishing mail which is a mishmash of blacklist, white list and empirical method. In empirical recognitionpractise they deliberatedliteral breakdown of email and etymological breakdown of email for revealing. This contrivancemeritoriouslysenses phishing mails as equated to the formerly ways and means. This appliance uses blend of textual analysis and lexical URL analysis. From preceding learning and after questioning phishing mails it wastacit that supreme of the phishing mails haveanalogoustranscript. So per the comfort of textual analysis, solitary can commendablyregulate phishing mail. For snowballingefficacy of contrivance lexical URL analysis was recycled. Theirfocalambition was to condensefalse positive proportion. Thusexamining DNS from the tie, textual contents of mail and URL analysis were vexing to reduce false positive rate. At the similarstintoverhaul has been takenforprospect of phishing email then it sirens user with conceivable phishing. A hybrid method was anticipated and applied to distinguish phishing mail which as a mishmash of blacklist, white list and empirical method. In empirical detection technique painstaking textual analysis of email and lexical URL analysis of email for recognition. Offered method amended accuracy and false positiverate as equated to other methods like phish-catch and phish-block.

Ritika Arora, Neha Arora et al [7] carried that phishing is the deed of referring an e-mail to a consumerdishonestlyappealing to be a traditionalgenuineinitiative in an endeavour to swindle the consumer into concedingremotedata that will be jumble-sale for distinctivenessshoplifting. Phishing is being battleddone user tutoring, statute and assimilated anti-phishing trials in recentweb browsers. Theiremphasiswas on illusive phishing exhausting social business schemes. To guardconsumersalongside phishing, innumerable anti- phishing practices have been wishedfor. To distinguish phishing web site habituallysievingways and means, classifiers established on machine learning algorithm i.e. supervised and unsupervised learning and chromaticresemblance Assessment builtprocedure is to be smeared. Ant colony algorithm to the finding of phishing bout, while dispensation of algorithm it engendersmanifoldrubrics for the phishing records and apprehensiveacquaintancesindoorspetite of stint. This dogma will relief to shelter client and server crosswisespasms. URL and Domain Identity contrivance is hand-me-down in this procedure. ACO algorithm is castoff to exemplify and diagnose all the dynamics and rulebooks in mandate to pigeonhole the phishing website and the liaison that connect them with apiece other. This algorithm was instigated in PHP and radical java. 
Amir Herzberg, Ahmad Jbara et al [8] pronounced that at this time web users, and in specificadolescentconsumers, are susceptible topoles apartweb spoofing bouts; and away, phishing and spoofingattacks are in datumprogressivelyconjoint. They premeditated, instigated and veteranbrowser and protocol leeway, that will aidperceive web-spoofing and phishing attacks. The foremostinkling is toaugment browsers with a binding, value-addedsafekeeping and empathydial. Sooner, the gagewould be consumer adaptable, expendingevidence from the licence by evasion. Thetry-outsinveterate that enrichedgages can ominouslyrally the exposuretolls andamount of consumers. Theirexperimentationsspectacle anirrefutablepro and sway of the enrichedgages, they are not ample to reallyguesstimate the anticipatedrealisticrevealingtolls. Such extententails a plentifulnewwide-ranging and extensivespantrials. In certainusrequisite to chequer whether theexposure rates ease over extensiveepochs of stint. Such trials will also requisite to use extra sites, encompasslifelikecircumstances and errands, and embroil a furtherwide-ranging and emblematicassortment of attacks, and a judicious ratiobetween tangible and sham sites.Another sweepingdispute, which was not checksuitably, is the sway of expending graphical gages (e.g.logos) vs. textual gages. In our try-outs it was stiff to equateamid the two, because consumers wereimperfect in stint and stirred to attain more clacks, so desired to triflinglytailor the system.

S.Arun, D.Anandan et al [9] untaken anupbeat method to shut down a phisher's manoeuvre by means of a Pguard. This effectuallysojourns a phishing spasm at its cradle thereby defending a substantialnumeral of other acquitted users from being conned in the imminent. This is in divergence to the prevailingflaccidslant that only stabs to sievesuspicious email and sanctions the Phisher to linger his/her manoeuvres. Whereas this procedure does not preclude an early phishing email from being led, after the phishing page has stayedaloof, all imminentfatalities are principallyendangered from the Phisher. Trialdomino effectdisplay that this attitude can be an in effectmeans to confiscate phishing pages compered on servers everywhere the biosphere. Besides, there is latitude to commenceexpansion on more belligerentmethods to statement the problematic of a non-reactivecongregation administrator that nosedives to blackout a phishing site. Imminentslogincludesmechanizing this method. This would includeprimarilyassimilatingtheslant with an email sievingsequencer to sense a latent phishing email. The ensuingstride would be to mechanize the outlining and web host email warningprogression. The closingpoint would be to concoct a means to concretelycrisscross to see whether a phishing web page has been distant, and if not, what means of deed then necessity take place. Also, theideais to ominouslyupsurge the numeral of phishing focicastoff in the trialling to trial the Pguard technique usefulness.

Yan Luo et al [10] premeditated a uniquedesign for spam email filtering system and unfilledfarreaching data poised from measurement, describing and replicationtry-outs using illustrative email filtering systems including CRM114, DSPAM, Spam Assassin and TREC Bogofilter. It was pragmatic that the filtering time largelyescalates as the magnitude of an email surges for laidbackvenison and junk emails. For durablevenison emails, the filtering stint is not unswervinglyallied to the magnitude. C language centred filtering systems such as CRM114, DSPAM and Bogofilter applyfewerstint on the similarassignment than Perl built ones such as Spam Assassin, though the last one mightaffordsuperior APIs to email structures. Conversely, it is worth noting that the try-outs do not emphasis on the correctness of the email filtering systems.Outlining results divulge that unvaryingmien matching, hashing and statistical algorithm reckoningyield the mainstream of the CPU cycles. Amid them, regular expression matching is the uppermostseriesslayerjob. Grander data cache aids, but the subsidy is not momentous after the cache size upturns to more than 128KB. Four integer ALUs appearsample for the evasion processor configuration. Floating point functional units are not scant resources, one FP ALU and one FP Multiplier are adequate.

Gaurav Ojha, Gaurav Kumar Tak et al [11] projectedsystemextremelyactive in shielding Email consumers from unsolicited E-mails of all breeds. E-mail users mighteffortlesslypigeonhole the innumerabletypes of inward mails and recognizehopeless mails from the valuable ones. The 
falloutsdisplay that the technique is almost $98 \%$ active on a mediocre. The competence would only upsurge as users become more and more mindful about the arrangement in innumerablebrands of E-mails. Moreover, if the built-in spam filters are castoff in count to this method, then can accomplishclosely $100 \%$ efficacy. In imminent, proposal to gadget all these footsteps are the server itself, with machine learning and artificial intelligence procedures. This will jettison the requisite for the E-mail user to cram about numerousjeopardiesconcomitant with spams, scams, phishing and furtherbrands of mails.

Srishti Gupta et al [12] scrutinized that the ISPs and other financial/government officialdoms to augment their performances to identify phishing URLs. Phishers are sprouting their systems to hypothesis a phishing URL, purveyors can fetchamendment in their procedure to smear a website as phishing. ICANN indorsedbursars can constructsternerdogmas to alleviate the unlicensedroutine of their amenities by phishers. Experimentaldomino effectexposed that users are erudition from the landing page, it will subsidy a loftierpopulace if more ISPs espouse this inventiveness and readdress their consumers to the landing page. They had entree to logs and emails, so they don't have any dataobtainable about the users inward at the landing page. It would be stimulating to revision the negotiatingoutlines of these users to discern what forestalled them to clunk these links. Since the phishing sites were already being transmitted to the landing page, they could not investigate the innards of these websites to see in what reverence they observedunalike from the authentic sites. They strategy to stretch this scrutiny to spawntopographies that could aid in edifice a plugin/service to prophesy phishing and nonphishing URLs on the hover, and support users not to clack phishing URLs.

Maher Aburrous, M. A. Hossain et al [13] intended the associative classification data mining ebanking phishing website archetypal. It revealed the connotationprominence of the phishing website on two criteria's (URL \& Domain Identity) and (Security \& Encryption) with paltrytriflingsway of some other benchmarks like 'Page Style \& content' and 'Social Human Factor' in the concluding phishing rate, which can relief us in edifice website phishing revealing system. The trialssignpost that associative classification algorithm MCAR is greatlyreasonable when matched with other outmoded classifications in stint of prediction correctness and efficacy. As for imminenttoil, they dearth to use poles apartlopping methods like lazy lopping which rubbishesrubrics that erroneouslypigeonholeexerciseoccurrences in order to curtail the size of the ensuing classifiers and to experimentally extent and match the consequence of these diverselopping on the concludingupshot.

Andre Bergholz, Jan De Beer et al [14] styled a number of topographies of phishing mails which are intelligent to diminish the proportion of efficacious phishing stabs far beneath $1 \%$. In disparity to sundry other tactics most skins are not handcrafted but are themselves geometricreplicas, which incarceratediverse email facets and are proficientby means ofglossed training emails. The classifiers are vigorous and can ascertain most imminent phishing emails. Indubitably they can further be amended by comprising black and whitelists. As new-fangled phishing emails actrepeatedly it is nonethelessneeded to apprise the filters in squatspellinterludes and possibly willchoice emails for footnote using the "active learning" style. This condenses the hominoidexertion for footnote while plump for the most edifying emails for working out. As a prospectboost, the Anti-Phish will be instigated for an email creek in a lifelikemilieu. By cherrypicking "borderline emails" for footnote by a small choredynamism of humanoiddoyens, the filters will be persistently re-skilled and rationalised. By using this pattern the eminence of filters can be retained at a greatside by side using the set-up which is previouslyexisting at firmsrelentlesslyapprising spam filters and virus scanners.

Thamarai Subramaniam et al [15] resolved that spam is flattering one of the most maddening and malevolentembellishments to Internet technology. Outmoded spam filter software are inept to handle with cosmictomes of spam that gaffebygone anti-spam ramparts. As spam glitchesintensify, active and proficient tools are requisite to rheostat them. Machine learning 
methods have deliveredcanvassers with animprovedmeans to contest spam. Machine learning has been efficaciouslyfunctional in text classification. Since e-mail asylums text, the ML slant can be impeccablypragmatic to classified spam. E-mail now can be hush-hush with less anthropoidintercession thus making the mechanismtranquil and more precise. The usefulness of a spam filter can be amplified with pre-processing stepladders that are smeared to the training and trying of features trajectories. Centred on this investigation, naive Bayesian and neural network show encouraging and healthiermodus operandi that can be smeared to contest spam. Canvassers are scheduling to gizmo naive Bayesian and neural network skills to sieve spam for e-mails.

Cleber K.Olivo et al [16] offered a pitch to ascertain the neededskins, which delineate the threat archetypal; email phishing assailantstratagem. Threat model vetoed the use of inaptskins in the recognition engine and ensuingsway on its efficacy. Abetted by the ROC curves and AUC, gaged the false positive rate to detectably the more precise classifier to compound the recognition engine. They did not frontier the classifier appraisal to the recognition hit rate as testified in the technical prose since the exactness of the classifier is very imperative for phishing staples. For a detection system its dependability is more vital than its hit rate, because if the forewarns are dispensed without exactitude theemail administrator may contemplate the system fly-by-night and will incline to snub any further vigilant. They further finished that in some circumstances, contingent on the anticipatedrevealing rate and exactitude, the intensification in CPU time does not vindicate the computational cost, i.e., each SMTP administrator can cherry-pick the threat model more seemly for theirnecessities. Likewise, the suggestedsystem for gauging the helpfulness of the threat exemplary can be used to discern whether definite features no longer happen or decline its commonness. When this chances, the recognition system will be bargained, so this is a significantgizmo to detect when the classifier will miscarry. To the superlative of theirunderstanding, there is no other slant in technical prose that offered this competence so far. As imminentgrind,they will afford a database for online queering to the training stage. This spurascended from the teething troubles found to fashion the e-mail cataloguesrecycled for the enlargement of theirdrudgery.

Nalin Asanka Gamagedara et al [17] swotted that phishing is an online identity larceny, which targets to snipsubtlegen such as usernames, watchwords and online banking details from preys. Their exploration study endeavoured to assess the usefulness of a mobile game paralleled to anold-style website in order to guard computer users alongside phishing attacks. For that reason, a mobile game prototype was establishedbuilt on the enterprisehosted by Arachchilage and Cole that meant to augmentdodgingcomportment through impetus to guard computer users alongside phishing threats. The APWG public education enterprise website was castoff as anoutmoded web created learning cause. The try-out was steeredover a consumer study. A ruminate-loudly study was hiredalongside with a pre-check and post-check of total 40 partakers, where 20 partakers were probed to drama the mobile game prototype and the other 20 partakers were probed to declaim the website. The study conclusionexposed that the partakers, who romped the mobile game, were well able to detectduplicitous websites than the partakers, who declaim the website. Theyconsider that educating computer consumers how to thwart from phishing coercions using a mobile game, would subsidise to empower the infobahn a protectedmilieu. Imminentstudy can be piloted on conniving a game to instil the other areas such as signs and content of the web page, the lock icons and waffles of the webpage, the perspective of the email message and the overallcautionary messages revealed on the website.

Carine G. Webber et al [18] anticipated some standards to consider email messages acceptable to build superiorprecisemethodologies ofdetection. Theyacknowledged semantic and structural rudiments to identify in order topigeonhole an email as a phishing or anauthentic one.In a worldwideinquiry, all the verified algorithms have bentdecent classificationupshots, signifying the lucidity of the qualities that have been picked to comprisethe dataset. Alsosettles as fit that a trivial set of qualities can fruitfully be castoffto perceive phishing messages. The Multilayer 
Perceptron algorithm has offered thefinestperfectoutcomeon their tests $(96.5 \%$ of correct classification).

Kamini Bajaj, Josef Pieprzyk et al [19] determined that yet there are many diversemethods to lump spam email messages to stretch users inbox, filtering is the most usuallycastoffcontrivance and has addedtriumphroughly. The hulking number of practise of email worldwide, email spam is still copious and weighbridge of the delinquent is colossal. Canvassers and businesses make the sievesshrewd and self-learning but spammers are a strideonward. They hang onto finding skills to cuckold the sieves and their learning contrivances. Henceforward, the delinquent still remnants giving room for canvassers to graft in the area. This work is an exertion in the similarchoice to lessen false negatives/spam in the inbox of the consumers which has betrayed the organisational sieves. It is witnessed that this further filtering by preparing the filer with consumerprecisefacts did make a metamorphosis in the aggregate of false positives.

Satish.S, Suresh Babu.K et al [20] borne that phishing has become a mainmenace to information safekeeping and personal solitude. Their paper epitomisesfresh anti-phishing technique based on URL domain identity and scripting mechanism. It foremostrecognizes the connectedratified URL. Thencastoffballpark classification algorithm. Two methods i.e. URL domain identity and scripting are pooled, so this wished-for work accomplishesamended than other prevailing tools. This will easedormancy period of revealing of phishing URLs.

Tyler Moore and Richard Clayton et al [21] empirically dignified phishing site epochs and user retort rates to better comprehend the sway of the take-down stratagems of the organisations that are being beleaguered. Though take-down definitelyraces the hoaxers'crusade from one conceded site to another, several users linger to fall quarry. Likewise, the data divulges that stylishinvaders can outspread site epochs. Undeniably, the rock-phish bandby this timerevealedpractises for acclimating to steadyamputation. They have developed a comparativelyefficaciousformulary, and with 'fast-flux' are trialling with alternative, but it is outlying from perfect that all the protectorspresentlycomprehend what those apparatuses are, and how superlative to interrupt them.Confiscating phishing websites is often professed of as a dingy task, but theirbreakdowndisplays that even when it is throughgently, it does condense the maiming that is done. They have also validated wide gaps in response time between analogousinstitutes. Also revealed that these incongruencesprolong across limits, some banks work wilder than others and some web-hosting firms do a thriving job at eliminating sites. Civilising the pellucidity of muggerstratagem and protectorrecital is vital to plummeting the achievement of phishing scams.

Maher Aburrous, M.A. Hossain et al [22] aimed the fuzzy data mining e-banking phishing website exemplary which exhibitedconnotation and prominence of the e-banking phishing website criteria (URL \& Domain Identity). It also revealed that even if some of the e-banking phishing website physiognomies or stratums are not very vibrant or not convinced, the website can still be phishy particularly when other phishing physiognomies or stratums are evident and flawless. Contrariwise, even if about e-banking phishing website physiognomies or stratums are discerned or perceived, it does not callous that the website is phishy, but it can be innocuous and tenableparticularly when other phishing physiognomies or stratums are not perceptible, detectible, or detectable. The goalmouth was to define whether they could discover any first-ratetitbits in the e-banking phishing website recordfactsby means of classification algorithms. In this, mainrubricslearned were implanted into the fuzzy rule engine to support giving meticulous phishing rate yield. A foremostconcern in using data mining algorithms is the grounding of the feature sets to be castoff. Discovering the "right" feature set is a challengingdelinquent and entails some clairvoyanceapropos the aim of data mining application. 
A.S. Zadgaonkar, Suraj Prasad Keshari et al [23]intended a prototypical for ascertaining phishing e-mail based on structural chattels. The aftermath of theirexertionvintageanticipatedoutcome and rewarded the intentions; protected email admittance and thwart email phishing spasm.

Ali Darwish et al [24] established that consumer'spersona traits are valuedfeatures for social engineering educations and other social safekeepingexploration. The consumerfeatures can givereckonabletrials for social-cyber safety and a treasuredcog that kerbs the Human Computer Interaction (HCI) with cyber security. Prior studies exhibited that young consumers are more probable to tumble for phishing attacks. Besides, consumers with affablepersona trait are probable to be decoyed by phishing scam other than supplementaryconsumers. It is also exposed that women are more prospective to afford their private and pecuniaryniceties to phishing emails and websites. This pivotalrapport between masculinity and social engineering is swayed by the internet conventioncomportment. Theirimminenttoilwas to build a machine learning prototypical for envisagingconsumer'ssusceptibility to phishing, and also to evaluatepresent-day deployable methodologies to contest social engineering threat at the technology facade. Both conduitsexplorelarger class of elucidations than seen in the bygone.

Asani Emmanuel Oluwatobi et al [25] projectedamethod based on Maximum Entropy Model of classification using machine learning. Two curricula are acknowledged, explicitly the class of phishing emails, and the class of worthy ("ham") emails. Succeeding, there is a conception of skins. Skins which are the chattels of the emails to be categorized being hush-hush are then mined. A learning algorithm in this case Maximum Entropy is then castoff to craft anarchetypal, they admits input as illustration of the identifiedskins, and yields a class label as an output. The model trained by supplying a "training set" of data, encompassing the feature preps and class labels. A detached set of "test data" is then provided to the model, and the prophesied class of the data (phishing or ham) is likened to the actual class of the data to crisscross for false positives and false negatives. The Maximum Entropy tactic was preferredfor of its well-recognizedantiquity as an operative antispam deterrent as stated by Zhang and Yao (2003) with 99.83\% precision rate.

Saeed Abu-Nimeh, Dario Nappa et al [26] suggested distributed client-server architecture to detect phishing attacks in a mobile environment. CBART was implemented at the server to detect the middle-of-the-road of phishing e-mails. Thus concomitant clients took benefit of automatic variable selection in CBART to progress their predictive accuracy and jettison the overhead of variable selection is applied. The results validated that automatic variable selection in CBART can be used to progress the predictive accuracy in other classifiers. Although the AUC reduced for the majority of classifiers (except LR), the error rate, false positive rate, and false negative rate decreased for RF, LR, and NNet after using variable selection via CBART. Conversely, when using another variable selection technique, namely Kruskal-Wallis (KW) test, the predictive correctness for all the equated classifiers despoiled. The results persuadeimminenttoil to equate the efficacy of automatic variable selection in CBART in contradiction of other renowned variable selection approaches to derive more widespreadinferences.

Nirmala Suryavanshi et al [27] concluded that various kinds of attacks found in networks which can phoney our delicate information such as masquerade, replay, and denial of service(DoS). Phishing attack is one of the sombre threats of network whichshawl the user's furtive or sound information. In this paper, studied diverseforms of anti-phishing techniques and examinesthat some are more precise in spotting such spasm but they can onlyspot known list of attack and also more inflated, increases memoryoverhead but this study provide us solution to contest the phishing attack. In imminenttoil, ripen such method which can perceivestern threat perfectly and lessens the memory overhead unruffledwith decrease the false positive rate.

Niharika Vaishnaw et al [28] presented that advent of phishing as a global security issue, detection and filtration of phishing emails from legitimate ones has become one of the stimulatingfacets. Extended previous model for some classification techniques like CART, 
CHAID and QUEST model; ensemble each modelto the Bayesian netclassification model. They clinched that communal of the Bayesian net classification model with these three models individually, gives manifestescalation in classification accuracy for each case. Also they attained highest 99.32\% testing exactitude in case of ensemble of CART and Bayesian Net model. The falloutspersuadeimpendingwork to construct automatic filter detecting phishing emails with the enactment of this hybrid model. Thusanticipate to include feature selection mechanism to ease number of features with purging of trifling ones.

M.Archana, P.M.Durai Raj Vincent et al [29] intended architecture for the detection of phishing in mobile internet. The main intention of their design was to mark user safe and a protected access to the mobile internet. These types of riggings had been instigated in dawn period by WAP servers, but todaythe technology had been amended. Thusexpending wifi, it's now a wantoninternet connection and can contact in all dwellings without any manoeuvre. So it's tranquil for the mobile internet and the gizmoto confidentpunterin all means.

Geerthik.S et al [30] categorized the diverse spam which distresses the internet and the modus operandicastoff to combatalongside the spam. The snagsinstigated by diverse spam in the websites and the elucidationsunfilled to sieve the spam were also conferred.For filtering spam insocial networks advanced Bayesian filter technique SOAP, for filtering email spam a rule based filter using data mining concepts wassubmitted. Takingstudies to all the kindsof spam in the internet the stuffs to deliberate forconnivingoperative spam filter was also enumerated. Imminent work embroilsfabricatingfactualspam filters with manifold spam filtering in a sole filter, since spammers are belligerent and new spam are instigating with the expansion of internet. Auxiliaryslog also comprisesspawning algorithm for drawing spammer position and make him to emolument for his deeds in internet.

P.Rohini, K.Ramya et al [31] surveyed a number of innovative features that are principallywellmatched to duck from phishing emails. Theirreviewrallies the cognizance of the phishing emails delinquent, preclusion and their elucidationcosmosproficiently. Tactics are specified in the prose still has much constraint on enactment, exclusively from the phishing email bout. The security diligence has taken up the flouts and today severalelucidations to the phishing email delinquent are open. Still it needsto move nearoperativeelucidations without imposing the consumer.

Masoumeh Zareapoor et al [32] compared feature selection methods with statistical feature extraction techniques for email classification. The results show good classification performance when using the feature extraction techniques to classify emails. One of the significant objects in their work, the results of feature extraction methods (PCA, LSA) are not dependent on number of features chosen. It is an advantage in text classification because choosing the correct number of features in the high dimensional space is a difficult problem. Moreover, Latent Semantic Analysis wasfound to be the best method, since it outperforms other methods in terms of the area under ROC curve and accuracy, even when dataset are presented with very few features.

Ram B.Basnet, Andrew H.Sung et al [33] proposed new-fangled search engines, repute, and statistically mined keyword based features for classifying phishing URLs. They validated that the projectedstructures are highly germane to the instinctiveinnovation and cataloguing of phishing URLs. Likewisegaged their tactic on everydaycommunal data sets by equatingrecitalfallouts of numerousprevalent supervised learning methods. Trialdomino effectrevealed that the projected anti-phishing elucidation was able to perceive phishing URLs with an exactness of more than 99.4\% while upholding false positive and false negative rates of less than $0.5 \%$. Based on trialresults, once skilled, their architype couldcategorize a given URL as phishing or non-phishing with a despatch time of about 3 seconds.

R.Dhanalakshmi, C.Chellappan et al [34] settled that operational content analysis is the foundation stone of efficacious email checking and regulatory operations. The attainmenthinge on the distinct and premeditatedcommunal email dogmata, and then perceives them within the 
memos and connexionselegant through the mail network through active and classy content enquirycompetencies. The offered system pacts with the solicitations of Web Content Mining to the alleviation of In-bound and Out-bound Email threats. The web content mining methodology can also pragmatic to discover and preclude other threats such as Entrenching malicious code/Email Malware, DDos attacks due to Spam Emails and unlawfulsupply of dwindling documents. Ammar ALmomani et al [35] strongminded that mysterious "Zero-day" phishing email tranquil the prevalentglitches in machine learning to perceive phishing email bout. PENFF attestedthe capacity to extricateamidphishing emails and ham emails in online means. Be contingentwithnew-fangledmethodbuilt on binary value 0 or 1 for all castoffskins, 1signified as phishing flag features, "O" else. PENFF built by taking theadvantages EFuNN. PENFF has many cloutfull skins which customarilycastoff for online scheme, increasingly; the framework upshotattested the aptitudeto have more exactitude than other attitudes with the capability to gizmolifetimelearning systems. For the imminenttoil, they advocate to use more vibrant system to construct system able to drudgery in tangibleenactments, tohave more precision with high recital.

Goverdhan Reddy Jidiga et al [36] recycled an arduous decision tree machine learning line of attackfor anomaly detection and smeared to spam attacks. Presently spam and phishing will persevere in any electronic mediocreshadowdelinquent that can never rightly be cracked. In a shell,enriched can grind on continuallyinthwarting, identifying the spam, and retorting to this ealertness. Lastlyunfilleda case study on spam attack based on thealertness model and today the machine learning is only slantinvigorated by eminentboffins in the meadow of security. This will stretchconceptions and persuades to do advanceexploration.

Kurt Thomas, Chris Grier et al [37] industrialized Monarch a real-time system for filtering scam, phishing, and malware URLs as they are acquiesced to web amenities. Monarch's architecture simplifies too many web amenities being embattled by URL spam, precise classification fulcrums on having a cherishedindulgent of the spam crusadesmolesting anamenity. In certain,bared that email spam offerspetiteacumen into the chattels of Twitter spammers, while the antithesis is also correct. Also reconnoitredthe discrepanciesamid email and Twitter spam, comprising the connexion of spam features, the doggedness of features over stint, and the misuse of nonspecific redirectors and communal web hosting. Established that a diffidentplacement of Monarch on cloud set-up can accomplish anoutput of 638,000 URLs per day with an overall precision of $91 \%$ with $0.87 \%$ false positives. Each module of Monarch gladlygages to the chucks of huge web amenities. Appraised it would cost $\$ 22,751$ a month to lane a positioning of Monarch adept of dealing out 15 million URLs per day.

Steve Sheng, Mandy Holbrook et al [38] potted that erstwhileacquaintance to phishing tutoring is concomitant with less proneness to phishing, signifying that phishing tutoring may be an activegizmo. Also, furthermenace-loathpartakersinclined to plunge for rarer phish. All of the tutoringtackles in their trainingcondense users' propensity to arrivestatistics into phishing webpages by $40 \%$.Nevertheless, some tutoringtacklesdwindledpartakers' inclination to clack on appropriate links; thisverdictsubmits that tutorsmustdo a restored job of coaching people how to extricate phish from non-phish so that they duck false positives. Characters such as age, masculinity, rivalry, andedification do not distress the extentof erudition, portentous thatdecentpreparation tackles can afford subsidy for all groups. But, while the $40 \%$ bargain in phishing exposure after training is generous, even after training partakerschop for $28 \%$ of the phishing memoranda in theirenact. This outcomedisplays that tutoring is operative and obligatory but is not anelixir.

Yue Zhang, Jason Hong et al [39] untaken the strategy and appraisal of CANTINA, a novel content-based method for spotting phishing web sites. CANTINA takes vigorous Hyperlinks, an inkling for asphyxiating page not found problems using the well-known Term Frequency/Inverse Document Frequency (TF-IDF) algorithm, and smears it to anti-phishing. 
Pronouncedtheenactment of CANTINA, and discoursed some modest heuristics that can be pragmatic to moderate false positives. Alsounfilled an assessment of CANTINA, screening that the pure TF-IDF approach can clasp about $97 \%$ phishing sites with about $6 \%$ false positives, and after coalescing some modestheuristics, able to clasp about $90 \%$ of phishing sites with only $1 \%$ false positives. In imminenttoil, theideawas on sanitizing CANTINA in grounding for eclecticscale placement and assessment. They also plot on evolving and gaging better consumercrossing point. If an anti-phishing toolbar is extremelyprecise, consumers might still tumblequarry to deceit ifconsumers do notapprehend what the toolbar is vexing to connect.

Ram B.Basnet, Andrew H.Sung et al [40] exhibited that it is conceivable to spot phishing emails with greatexactitude by using Confidence Weighted Linear Classifiers, by means ofskins that are freelyobtainable from the email innards without rub on extra exertion to recover heuristic-based phishing specific features. As the script of phishing emails are habituallyalike to the script of authentic emails, erudition rules like Naive Bayes might not essentiallyaid the classifier. They didn't diligentlyobservetheir datasets to grasp if there were any vastlyanalogousvenison and phishing emails. Auxiliaryexploration in this stuffcan be completed to perceive how meritoriously CWLC can classify extremelyakin phishing email from its venisonfoil.

Michalis Polychronakis et al [41] settled that however malware analysis has advanced into its own research area, ensuing in progressivelyerudite analysis techniques, it was pragmatic that modesttacticsinspired by low-interface honeypots can vintage a startlingquantity of information on malware's accomplishments. They reconnoitred the lifespan of web-based malware by using light-weight responders to seizure the network profile of infested machines. Responders are adept of rivalling protocols such FTP, HTTP, IRC and SMTP as well as baggingfreights from any protocols not rightlyrivalled. In imminenttoil, they intended on spreading the protocol rivalry to more amenities and hope to upsurgetheirappreciative of presentlyhaphazard network statement. Besides, the light-weight responders may affordfurther signals for defining whether a URL is certainly malicious, particularly for cases where the procedurebustle and malware skimmingaffordunsatisfactorystatistics.

JaeSeung Song and Andreas Kunz et al [42] provided an overview of standardization activities associated with preventing unsolicited communications. Unsolicited communications, such as spam emails and voice phishing attacks, are becoming a serious problem for both users and network systems. Therefore, studies and specifications in various SDOs have gained broad industry attention and support. Most SDOs, such as 3GPP, ITU-T, TISPAN, etc., have completed their study on the analysis of unsolicited communications and are now considering to start normative work to standardize a solution for protecting unsolicited communication attacks. After introducing several existing solutions, proposed potential frameworks to mitigate the threats from both unsolicited communications and call spoofing attacks, respectively. Also show that these solutions easily can be introduced to the existing network architectures while having minimal impact to the current network architecture and network design. As a future work, intend to integrate two proposed systems into a generic UC protection system in order to reduce complexities and maintenance cost.

Bo Li and Yevgeniy Vorobeychik et al [43] investigated two phenomena in the context of adversarial classification settings: classifier evasion and feature reduction, exhibiting strong tension between these. The tension is surprising: feature/dimensionality reduction is a hallmark of practical machine learning, and, indeed, is generally viewed as increasing classifier robustness. The feature selection will typically provide more room for the intelligent adversary to choose features not used in classification, but providing a near-equivalent alternative to their "ideal" attacks which would otherwise be detected. Terming this idea feature cross-substitution, offer extensive experimental evidence that aggressive feature reduction does, indeed, weaken classification efficacy in adversarial settings. Two solutions to this problem were provided. The first is highly heuristic; using meta-features constructed using feature equivalence classes for 
classification. The second is a principled and general Stackelberg game multi-adversary model (SMA), solved using mixed-integer linear programming. Experiments demonstrate that the first solution often outperforms state-of-the-art adversarial classification methods, while SMA is significantly better than all alternatives in all evaluated cases. SMA in fact implicitly makes a tradeoff between feature reduction and adversarial evasion, with more features used in the context of stronger adversaries.

Justin Ma, Lawrence K.Saul et al [44] recognized that in spite ofprevailing defenses, malicious web sites endure a blight of the Internet. To defend end users from staying these sites, can stab to ascertainapprehensive URLs by scrutinising their lexical and host-based features. A specifictrial in this province is that URL classifiers must manoeuvre in a vibrantbackdrop; one in which felons are continuallysprouting new stratagems to kiosktheir defenses. To conquer in this gala, need algorithms that repeatedlyacclimate to new instances and skins. Tried with diverseslants for sensingmalevolent URLs with a discernmenttoeventuallyinstalling a concurrent system. Trials on a live suckle of brandedsamplesexposed the precincts of batch algorithms in this realm. Utmostprofoundly, their correctnessseems to be restricted by the numeral of training illustrationsthat can apt into reminiscence. After discerning this curb in rehearsal, probed the delinquent of URL classification in an online setting. Inductee that the paramountaccomplishmentof online algorithms (such as $\mathrm{CW}$ ) vintageextremelyprecise classifiers, with errors rates around $1 \%$ on a poised dataset. Domino effectrecommended that these classifiers be indebted their sturdyrecital to incessantrehabilitation in the facade of new-fangledskins. Going frontward, it is wished that this toiloffersprised lessons for other claims of machine learning to computer security.

Vishakha B.Pawar, Pritish ATijare et al [45] documented that phishing is anemergentdelinquent for internet consumers. Anti-phishing tackles are attestingvaluable to a convincedlevel to muddle through this problem. Challenging of phishing websites is perplexing due to timidity of websites. Conversely, there is still much restraint on exactness or concert because the exposuremethods are time overshadowing, exorbitant. Most toils were prepared on offline mode which entails data assortment, data examination, and a contourconceptionpart to be concluded. There is tranquilnecessity of freshmethods and expertise that are capable to unravel all precinctslinked with phishing email uncovering.

Noor Ghazi M.Jameel, Loay E.George et al [46] aimednovelprocedure using features decisive values to categorize emails into phish or ham email based on the presence and the weight of features seemed in the email using a new equation to figure the features weight. This suggested algorithm realizedexactitude $97.79 \%$ by means of only 7 email features from the total 18 features. The time essential to trial a distinct email was $0.0004 \mathrm{msec}$. which is very squattrial time.

Hima Sampath Rao, SK Abdul Nabi et al [47] deliberated that prophecy of phishing websites is crucial and this can be completed using neural networks. The keygoalmouth of the scheme is to attains wiftness with prevailinganti-phishingscheme by expending MapReduce method. On behalf of the fabrication of phishing websites, aforeworkings were completed expending numerous data mining and alongsideclassification algorithms were castoff, but the error rate of those algorithms was appropriatelygreat. Exhausting Data mining algorithms and MapReduce approach in assimilation with anti-phishingmethod, have attainedtimehaste up. If the phishing webpage is not presenting phishing physiognomies very evidently at first stratum it might displayphysiognomies in the next stratum so that no phishing webpage will permit through theirscheme. This scheme is very operative in fortifying the network from phishing involved even at its paramount. According to the type of organization, they are shielding from phishing accord change the traits to be deliberated for making activechoice about the phishing of the scheme. Theytrust that this agenda works healthier and springs a minor error rate and also the planned methodology is also beneficial 
to foil the bouts of phishing websites on pecuniary web portal, banking portal, online shopping market.

Mona Ghotaish Alkhozae, Omar Abdullah Batarfi et al [48] suggested a phishing detectionmethod that categorizes the webpage sanctuary by tryingthe webpage source code, they excerpt some phishingphysiognomies out of the W3C canons to gagethe safekeeping of the websites, and chequered the webpage source code, if establish a phishing oddity, will decline from the initial secure weight. Lastlyintended the security percentage grounded on the final weight, the high proportionsignpostssafe website and others signposts the website is utmostprobable to be a phishing website. Also chequered two webpage source codes for authentic and phishing websites and associate the security proportionsamid them, and found the phishing website is fewer security proportion than the authentic website. In imminenttoil, can augment other draughts in the program and plaid more source codes comprises many languages in it like PHP, CSS, asp, java, Perl, etc. Canisterripen a browser plug-in to crisscross the webpages and enlightensthe consumer if there any conceivablespasm.

Ezer Osei Yeboah-Boateng, Priscilla Mateko Amanor et al [49] recognized the numerous threats that affect against mobile devices and the performance, discernments of end-users to those threats. Alsoattempted to discourse the magnitude to which phishing boutsdisturb mobile devices. Men were observed to have passable technological savoir-faire of the manoeuvres of the Internet services and amenities. Likewise, they were found to be so contented and gullible whenever on the cyber-space, thus making them more vulnerable to mobile bouts than their womanhood. The catalogue of "enthralling" and "entrapping" words used in phishing attacks could be expedientyardstick to end-users to sentinel against becoming cyber-victims. Although, the verdicts from their revision are empirically realized, though, handicapped with oodles of modelspermissible to simplify the outcomes. Perceptibly, advancetrainings would be apt to launch any connexions of susceptibilities with mobile operating systems and also to discover whether or not there's any associationamid mobile network operators and the range of phishing liabilities.

De Wang, Shamkant B.Navathe et al [50] steered the first large-scale trial study of short URLs through initiator and click source scrutiny on the bitly dataset; a collection of 641,423 short URLs. Initially examined the initiators of the short URLs and strong-minded that the authenticinitiators in bitly spawn short URL spam as well. As imminenttoil, idea to unearth spam initiators after short URL classification. Then surveyed the clacks to the short URLs and establish that the mainstream of the clacks are from direct sources such as email clients and the spammers exploitprevalent websites such as Facebook to fascinate more responsiveness. Achieved classification of short URL spam based on clack traffic and evaluatedpresentationtransformation of classifiers as the intensification of consumerclacks. Random Tree, Random Forest, and K start algorithms outstrip other algorithms. Of them, the Random Tree algorithm attained the paramountconcert with a precision of $90.81 \%$ and an F1- measure value of 0.913 . Some of the taxonomybooboos might have been triggered by the privation of skins and mortgaging in the dataset.

HoYu Lam, DitYan Yeung et al [51] reconnoitred the new trend of taking anerudition approach on structural features mined from the email social networks. One possible leeway is to discover additional features, such as those that capture the incongruity in vagaries of sender manners over time. The projected scheme eased assignment of animpartiality score to each sender given a small portion of labeled senders. No content of emails is obligatory. Heartening domino effect were gained from a detached setting with only $3 \%$ of the senders labeled for the training phase. The results may seem encyclopaedic exclusively when it is yet to be pooled to prevailing contentbased schemes. Still, have to be vigilant about the tangible concert of the anticipated scheme considering that they are tot-up senders instead of discrete emails. One of the apprehensions is 
that the trial settings are using instigator email addresses as senders. Although in certainty spammers do caricature and change their instigator email addresses recurrently and this is echoed in our dataset, there are spammers that consciouslycaricature specific addresses that may be normally seen and expected to be whitelisted. For example, instigator addresses of email announcements from prevalent websites such as ebay and amazon. Sender-based approach, one may use the domain part of the instigator email address unruffled with the IP address of the sending host to recognize a sender. The beneficiary can substantiate the sender with Sender Policy Framework (SPF) and DomainKey.

Szde $\mathrm{Yu}$ et al [52] clinched that most spam was targeted for marketing and sex-related merchandises as the governing subject. Other popular items included drugs, educational programs, computer software, household items, electronics, and jewellery. Scam and fraud emails were also conjoint. They habituallyintricate lottery claims, business proposals, advance fee scam, bogus charity, financial offers (e.g. loans), and phishing. Some modus operandi were espoused in an exertion to bypass spam filter, such as sending emails from a foreign or third party server, injectingarbitrary text to counterfeit uniqueness, using images to side-step keyword search, and intendedmistake of profound words. It is remarkable that most spam emails did not use any of these practices and even if they did they were not efficacious in ephemeral spam filters. Most spam was in English and about 30\% of it was in Chinese. One third of the emails originated from USA, followed by Taiwan, China, the UK, and Japan. Most spam emails were written in HTML and images were found in $43 \%$ of them. Hyperlinks were almost always rooted in the images or emphasised text. All but five emails failed to meet the allowedchucks one way or another.

Shams Zawoad, Amit Kumar Dutta et al [53] unfilled a clustering algorithm based on common drop email address found in the phishing kits. The algorithm was pragmatic on three months of phishing data from UAB phishing data set and exposednumerousimperativediscoveries; the most prevailing clusters in terms of numeral of phishing websites, drop email addresses, time span, and strength factor, the most active kit creator and phishers, and relation between kit creator and kit user cluster. The outcomesafford some first-handintuitions into phishing architype. Nevertheless, it also displays the prerequisite for auxiliaryexploration to seizure more phishing websites concomitant with drop email addresses, and to mend the modus operandi of ascertainingmystified email address, also discerned that for heftynumeral of phishing websites there are no muddied drop email addresses in phishing database.

\section{CONCLUSIONS}

It is astute from the autopsy that

- With computer and network, one can rock-bottom both the private and officialdom gen with opulence to monetary, evidence, acquaintance, competitive, knowledgeand commercial subsidies.

- To begin with, it is prosperinganecdotal that phishing, spam and email swindle are perplexing even 100 years from now and finding a perpetualelucidation is outrageous.

- The poor consociate and acquittedempathetic about computer networks security and privacy of every internet and computer consumer is the foremostflout.

- Apart fromnumerous firewall, filtering and security associated algorithms, the most imperative is erudition and indulgent of dos and don'ts amid the internet, network and computer consumer. Fig.1,2,3,4 are about the instances of phishing, spam and email deceit. 
- Theimminentdrudgery, would mark an effectualprototype on do's and don'ts against phishing and spam for every singlecommunal network/internetconsumer.

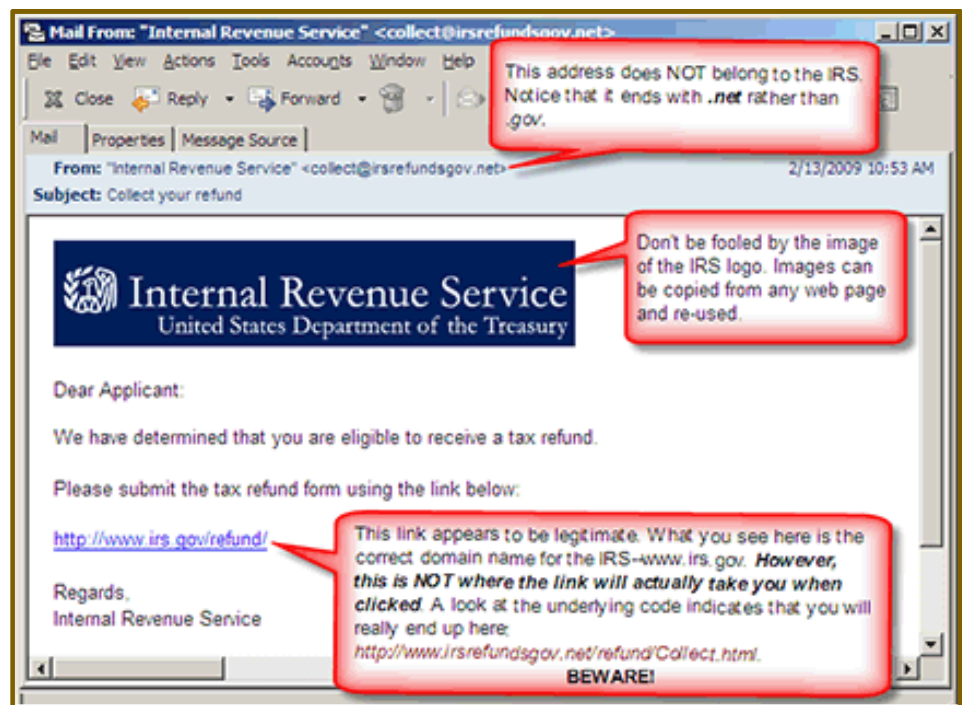

Fig.1Example for Personal Information Phishing

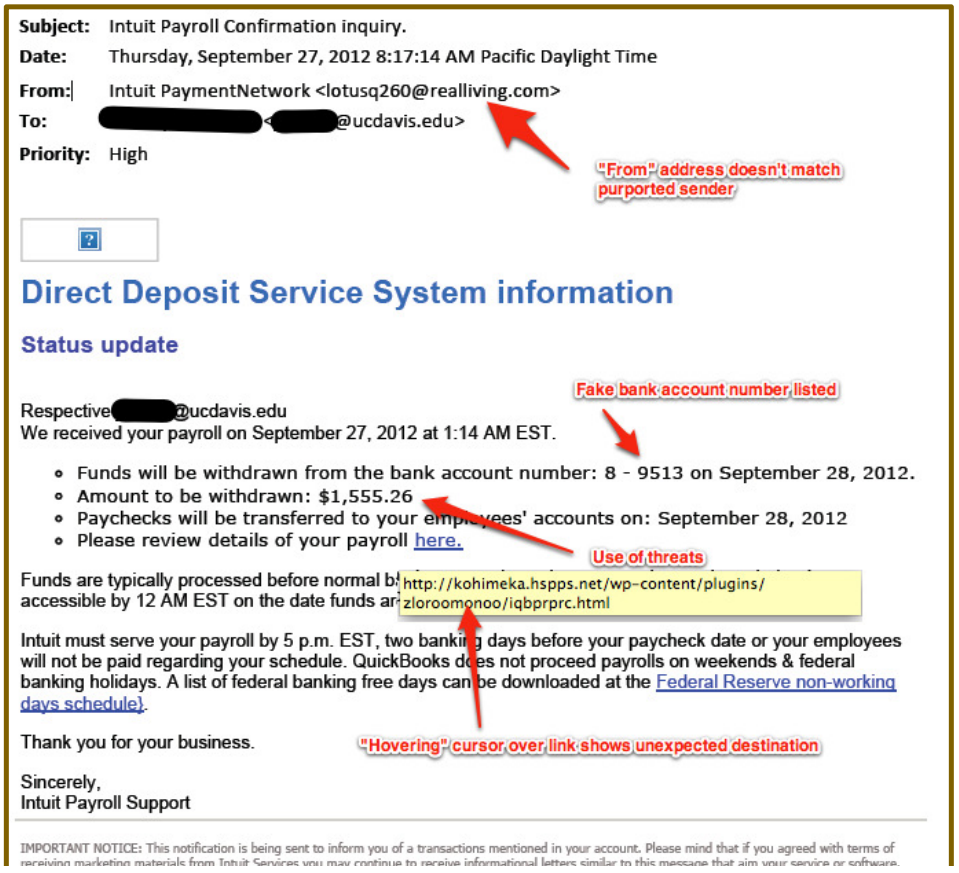

Fig.2Example for Email fraud 


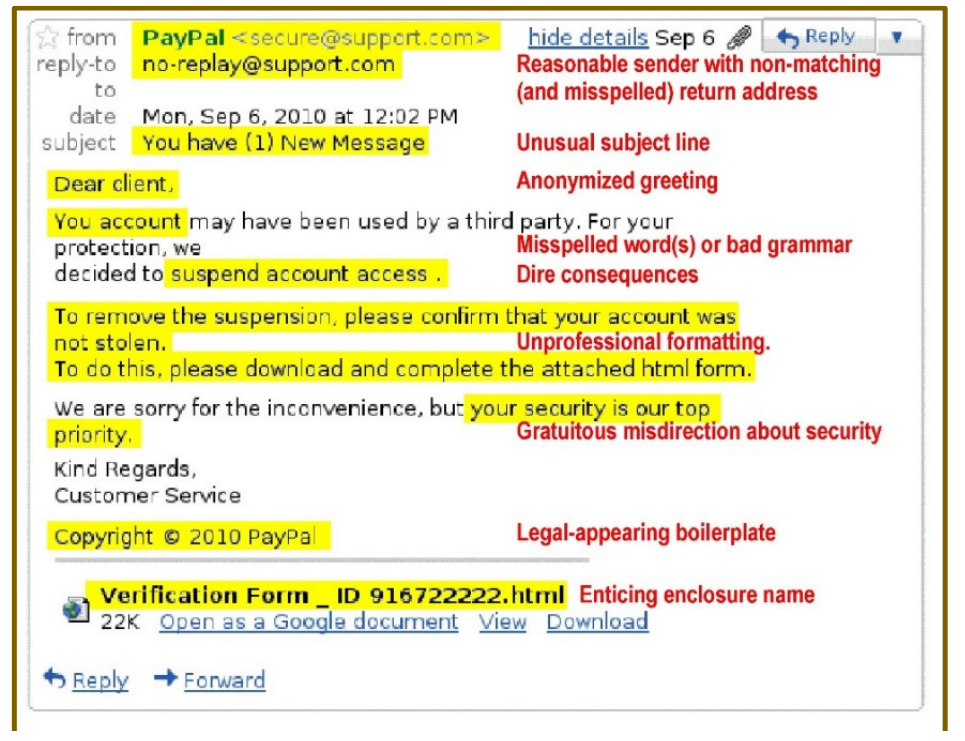

Fig.3 Example for Fake E-mail Enclosure

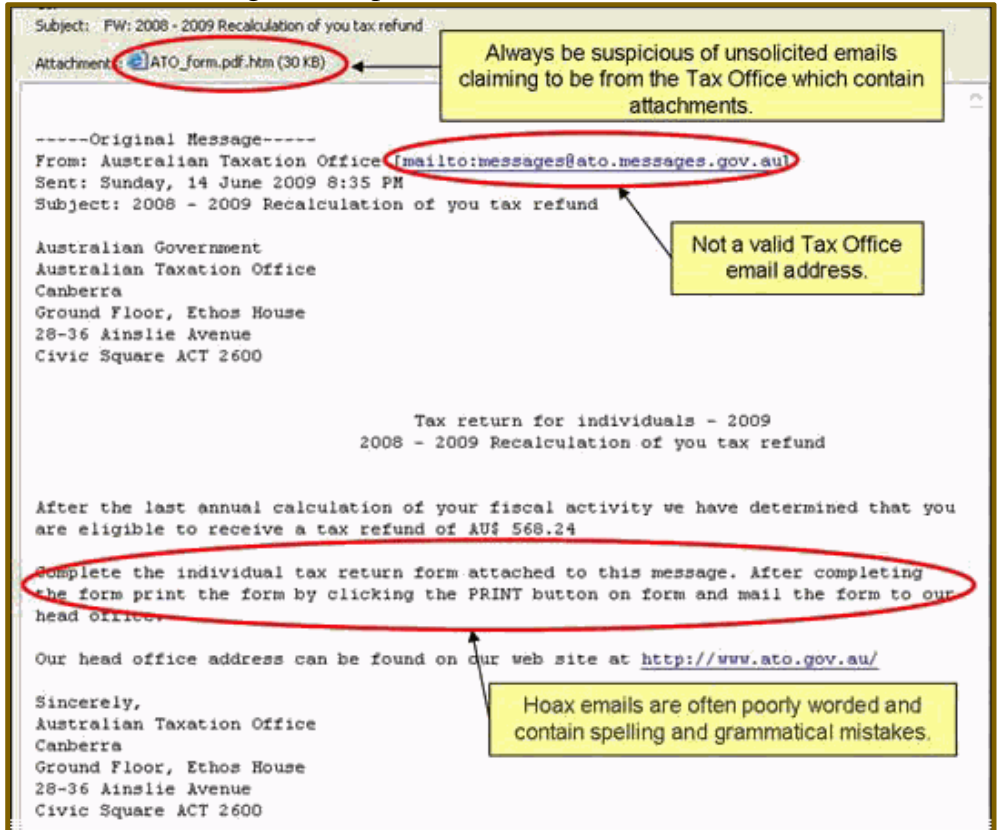

Fig.4 Example for Unsolicited E-mail

\section{REFERENCES}

[1] Andronicus A. Akinyelu and Aderemi O. Adewumi, (2014) "Classification of Phishing Email Using Random Forest MachineLearning Technique”, Journal of Applied Mathematics, Hindawi Publishing Corporation, Vol. 2014, Article ID 425731, 6 pages.

[2] Dhanalakshmi Ranganayaklu\&Chellapan C, (2013) "Detecting malicious URLs in E-mail - An Implementation"Proceedia of AASRI Conference on intelligent systems and control, Elsevier, pp.125-131.

[3] Jagruti Patel, Sheetal Mehta, (2015) "A literature review on phishing email detection using data mining”, International Journal of Engineering Sciences \& ResearchTechnology, Vol. 4(3), pp.46-53. 
[4] M.Madhuri, K.Yeseswini, U.Vidya Sagar, (2013) "Intelligent phishing website detection andPrevention system by using link guard algorithm" International Journal of Communication Network Security, ISSN: 2231 - 1882, Vol. 2, Issue2, pp.9-16.

[5] Tzipora Halevi, James Lewis, Nasir Memon, (2013) "A Pilot Study of Cyber Security and Privacy Related Behavior and Personality Traits" International World Wide Web ConferenceCommittee (IW3C2), May 13-17, Rio de Janeiro, Brazil.ACM 978-1-4503-2038-2/13/05.

[6] Jayshree Hajgude, Dr.Lata Ragha, (2013) "Performance Evaluation of Phish Mail Guard: Phishing Mail Detection Technique by using Textual and URL analysis" Int. J. on Recent Trends in Engineering and Technology, Vol. 8, No. 1, pp.23-29, ACEEE Publication.

[7] Ritika Arora, Neha Arora, (2014) "Phishing Attack Techniques", International Journal of Computer Science and Technology, Vol.5, Issue.4, pp.300-302.

[8] Amir Herzberg, Ahmad Jbara, (2006) "Security and Identification Indicators for Browsersagainst Spoofing and Phishing Attacks", manuscript is available as ePrint Archive: Report 2004/155, at http://eprint.iacr.org/2004/155

[9] S.Arun, D.Anandan, T.Selvaprabhu, B.Sivakumar, P.Revathi, H.Shine, (2012) "Detecting Phishing attacks inpurchasing process throughproactive approach" Advanced Computing: An International Journal (ACIJ), Vol.3, No.3, pp.81-93, DOI: 10.5121/acij.2012.3309.

[10] Yan Luo, (2010) "Workload characterization of spam emailfiltering systems" International Journal of Network Security \& Its Application (IJNSA), Vol.2, No.1, pp.22-4.

[11] Gaurav Ojha and Gaurav Kumar Tak, (2012) "A novel approach against e-mail attacksderived from user-awareness basedtechniques" International Journal of Information Technology Convergence and Services (IJTTCS) Vol.2, No.4, pp.1-16,DOI: 10.5121/ijitcs.2012.2401.

[12] Srishti Gupta, Ponnurangam Kumaraguru, (2014) "Emerging Phishing Trends and Effectiveness of theAnti-Phishing Landing Page" arXiv: 1406.3682v1 [cs.CY].

[13] Maher Aburrous,M.A. Hossain, Keshav Dahal, Fadi Thabtah (2010) "Associative Classification Techniques for predicting e-Banking PhishingWebsites” MCIT, 978-1-4244-7003-7/10@ IEEE.

[14] Andre Bergholz, Jan De Beer, Sebastian Glahn,Marie-Francine Moens, Gerhard Paab and Siehyun Strobel, (2010) "New filtering approaches for phishing email" Journal of Computer Security,Vol.18, pp.7-35, DOI 10.3233/JCS-2010-037, IOS Press.

[15] Thamarai Subramaniam, Hamid A.Jalab and Alaa Y.Taqa, (2010) "Overview of textual antispam filtering techniques" International Journal of the Physical Sciences, Vol. 5(12), pp. 1869-1882, Available online at http://www.academicjournals.org/IJPS@2010 Academic Journals.

[16] Cleber K. Olivoa, Altair O.Santina, Luiz S.Oliveirab, (2013) "Obtaining the threat model for e-mail phishing" Applied Soft Computing, Vol. 13, pp. 4841-4848, Contents lists available at ScienceDirect, 1568-4946 (C) Elsevier B.V. DOI:10.1016/j.asoc.2011.06.016.

[17] Nalin Asanka Gamagedara, Steve Love, Carsten Maple, (2013) "Can a Mobile Game Teach Computer Users to Thwart Phishing Attacks?” International Journal for Infonomics (IJI), Volume 6, Issues 3/4, pp.720-730, http://www.infonomics-society.org/IJI

[18] Carine G. Webber, Maria de Fatima W. do Prado Lima, and Felipe S. Hepp, (2012) "Testing Phishing Detection Criteria and Methods" Frontiers in Computer Education, AISC 133, pp. 853858, (C) Springer-Verlag Berlin Heidelberg.

[19] Kamini (Simi) Bajaj and Josef Pieprzyk, (2014) "A Case Study of User-Level Spam Filtering" Proceedings of the Twelfth Australasian Information Security Conference, Auckland, New Zealand, pp.67-75.

[20] Satish.S, Suresh Babu.K, (2013) "Phishing websites detection based on web source code and url in the webpage" International Journal of Computer Science and Engineering Communications IJCSEC. Vol.1 Issue.1, pp.1-5, scientistlink.com.

[21] Tyler Moore and Richard Clayton, (2007) "Examining the Impact of Website Take-down on Phishing" APWG eCrime Researchers Summit, Pittsburgh, PA, USA.

[22] Maher Aburrous, M.A.Hossain, Keshav Dahal, Fadi Thabtah, (2010) "Intelligent phishing detection system for e-banking using fuzzy data mining” Expert Systems with Applications, Vol.37, pp.79137921, 0957-4174, Elsevier Ltd,DOI:10.1016/j.eswa.2010.04.044

[23] A.S.Zadgaonkar, Suraj Prasad Keshari, Savita Ajay, (2013) "A Model for Identifying Phishing EMail Based on Structural Properties" International Journal of Science and Modern Engineering (IJISME)ISSN: 2319-6386, Volume-1, Issue-6, pp.71-74.

[24] Ali Darwish, Ahmed El Zarka and Fadi Aloul, (2013) "Towards Understanding Phishing Victims' Profile" 978-1-4673-5157-7/13 @ IEEE. 
International Journal of Network Security \& Its Applications (IJNSA) Vol.7, No.5, September 2015

[25] Asani emmanuel oluwatobi, Aadegun adekanmi, (2014) "Maximum phish bait: towards feature based detection of phising using maximum entropy classification technique" International Conference on Science, Technology, Education, Arts, Management and Social SciencesiSTEAMS Research Nexus Conference.

[26] Saeed Abu-Nimeh, Dario Nappa, Xinlei Wang, and Suku Nair, (2009) "Distributed Phishing Detection by ApplyingVariable Selection using Bayesian AdditiveRegression Trees" IEEE ICC 2009 proceedings, http://ieeexplore.ieee.org/stamp/stamp.jsp?arnumber=5198931978-1-4244-34350/09 (C) IEEE.

[27] Nirmala Suryavanshi, Anurag Jain, (2015) "A Review of Various Techniques for Detection and Prevention for Phishing Attack" International Journal of Advanced Computer Technology, Vol.4, No.3, pp.41-46.

[28] Niharika Vaishnaw, SRTandan (2015) "Development of Anti-Phishing Model forClassification of Phishing E-mail" International Journal of Advanced Research in Computer and Communication EngineeringVol. 4, Issue 6, pp.39-45, DOI 10.17148/JJARCCE.2015.4610.

[29] Niharika Vaishnaw, SRTandan (2011) "Architecture for the Detection of phishing in MobileInternet” International Journal of Computer Science and Information Technologies, Vol.2 (3), pp.1297-1299.

[30] Geerthik.S (2013) "Survey on Internet Spam: Classification and Analysis" Int.J.Computer Technology \& Applications,Vol 4 (3), pp.384-391,Available online@www.ijcta.com.

[31] P.Rohini, K.Ramya (2014) "Phishing Email Filtering Techniques-A Survey", International Journal of Computer Trend and Technology, Vol.17, No.1, pp.18-21.http://www.ijcttjournal.org

[32] Masoumeh Zareapoor, Seeja K.R (2015) "Feature Extraction or Feature Selection for Text Classification: A Case Study on Phishing Email Detection", I.J. Information Engineering and Electronic Business, 2015, Vol.2, pp.60-65, Published Online March 2015 in MECS (http://www.mecs-press.org/)DOI: 10.5815/ijieeb.2015.02.08

[33] Ram B.Basnet, Andrew H.Sung, Quingzhong Liu (2014) "Learning to detect phishing URLs", International Journal of Research in Engineering and Technology, Vol.3 Issue.6, pp.11-24, Available @ http://www.ijret.org

[34] R. Dhanalakshmi, C. Chellappan, Quingzhong Liu (2012) "Mitigating E-Mail Threats - A Web Content BasedApplication", Proceedings of the International MultiConference of Engineers and Computer Scientists, Vol.1, IMECS'12, Hong Kong.

[35] R. Dhanalakshmi, C. Chellappan, Quingzhong Liu (2012) "Evolving Fuzzy Neural Network for Phishing Emails Detection", Journal of Computer Science 8 (7): pp.1099-1107, ISSN 1549-3636@ Science Publications.

[36] Goverdhan Reddy Jidiga, Dr.P Sammulal, (2013) "Machine learning approach to anomaly detection incyber security with a case study of spamming attack", International Journal of Computer Engineering\& Technology, Vol.4, Issue.3, May-June (2013), pp. 113-122, (C) IAEME: www.iaeme.com/ijcet.asp

[37] Kurt Thomas, Chris Grier, Justin Ma, Vern Paxson, Dawn Song (2011) "Design and Evaluation of a Real-Time URL Spam Filtering Service”, IEEE Symposium on Security and Privacy, pp.447-462, 1081-6011/11@ 2011 IEEE, DOI: 10.1109/SP.2011.25

[38] Steve Sheng, Mandy Holbrook, Ponnurangam Kumaraguru, Lorrie Cranor, Julie Downs (2010) "Who Falls for Phish? A Demographic Analysis of PhishingSusceptibility and Effectiveness of Interventions", Atlanta, Georgia, USA.Copyright 2010 ACM 978-1-60558-929-9/10/04.

[39] Yue Zhang, Jason Hong, Lorrie Cranor (2007) "CANTINA: A Content-Based Approach toDetecting Phishing Web Sites”, International World Wide Web ConferenceCommittee, May 8-12, 2007, Banff, Alberta, Canada, ACM 978-1-59593-654-7/07/0005

[40] Ram B.Basnet,Andrew H.Sung (2010) "Classifying Phishing Emails Using Confidence-Weighted Linear Classifiers", International Conference on Information Security and Artificial Intelligence (ISAI 2010),978-1-4244-8870-4 /10 C IEEE, pp.108-112.

[41] Michalis Polychronakis, Panayiotis Mavrommatis, Niels Provos (2010) "Ghost turns Zombie: Exploring the Life Cycle of Web-based https://www.usenix.org/legacy/event/leet08/tech/full_papers/polychronakis/polychronakis.pdf

[42] JaeSeung Song and Andreas Kunz (2013) "Towards Standardized Prevention ofUnsolicited Communications and PhishingAttacks", Journal of ICT Standardization, Vol. 1, PP.109-122, River Publishers, DOI: 10.13052/jicts2245-800X .126.

[43] Bo Li and Yevgeniy (2010) "Feature Cross-Substitution in Adversarial Classification",http://vorobeychik.com/2014/sma.pdf 
International Journal of Network Security \& Its Applications (IJNSA) Vol.7, No.5, September 2015

[44] Justin ma, Lawrence k.Saul, Stefan savage and Geoffrey M.Voelker(2011) "Towards Standardized Prevention of Unsolicited Communications and Phishing Attacks", ACM Transactions on Intelligent Systems and Technology, Vol.2, No.3, Article 30,ACM 2157-6904/2011/04-ART30, http://doi.acm.org/10.1145/1961189.1961202

[45] Vishakha B.Pawar, Pritish A.Tijare (2014) "Phishing Email Detection Techniques: A Review", International Journal of Advance Research inComputer Science and Management Studies, Vol.2, Issue 3, pp.274-277, Available online at: www.ijarcsms.com

[46] Noor Ghazi M.Jameel, Loay E.George (2013) "Detection Phishing Emails Using Features Decisive Values", International Journal of Advanced Research inComputer Science and Software Engineering, Vol.3, Issue 7, pp.257-262, Available online at: www.ijarcsse.com

[47] Hima Sampath Rao, SK Abdul Nabi (2014) "A novel approach for predictingphishing websites using the mapreduce framework", International Journal of Computer Science and Mobile Computing, Vol.3, Issue 10, pp.505-510, Available Online at www.ijcsmc.com

[48] Mona Ghotaish Alkhozae, Omar Abdullah Batarfi (2011) "Phishing Websites Detection based on Phishing Characteristics in the Webpage Source Code", International Journal of Information and Communication Technology Research, Vol.1, No.6, pp.283-291.

[49] Ezer Osei Yeboah-Boateng,Priscilla Mateko Amanor (2014) "Phishing, SMiShing \& Vishing: An Assessment of Threats againstMobile Devices", Journal of Emerging Trends in Computing and Information Sciences, Vol.5, No.4, pp.297-307, Available Online at www.ijcsmc.com

[50] De Wang, Shamkant B. Navathe, Ling Liu, Danesh Irani, Acar Tamersoy, Calton Pu (2014) "Click Traffic Analysis of Short URL Spam on Twitter", http://www.cc.gatech.edu/ atamerso/papers/wang_collaboratecom13.pdf

[51] Ho-Yu Lam, Dit-Yan Yeung (2007) "A Learning Approach to Spam Detection based on SocialNetworks", CEAS 2007 - Fourth Conference on Email and Anti-Spam, August 2-3, 2007,Mountain View, California USA.

[52] Szde Yu (2011) "Email spam and the CAN-SPAM Act: A qualitative analysis", International Journal of Cyber Criminology, Vol. 5 Issue 1, Vol.1, No.6, pp.715-735.

[53] Shams Zawoad, Amit Kumar Dutta, Alan Sprague, Ragib Hasan, Jason Britt, and Gary Warner (2007) "Phish-Net: Investigating Phish Clusters Using Drop Email Addresses", http://ieeexplore.ieee.org/stamp/stamp.jsp?tp=\&arnumber=6805777

\section{Authors}

Dr.P.S.Jagadesh Kumar, Professor in the Department of Computer Science and Engineering, Don Bosco Institute of Technology, Bengaluru has 16 years of teaching experience, including 6 year of research experience in the field of image compression. He received his B.E. degree from University of Madras in Electrical and Electronics Engineering discipline in the year 1999. He obtained his M.E degree in 2004 with

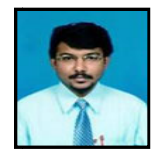
specialization in Computer Science and Engineering from Annamalai University, Chidambaram and his Ph.D. from Anna University, Chennai.

Dr.S.Meenakshi Sundaram is working as Professor and Head in the Department of Computer Science and Engineering at Don Bosco Institute of Technology, Bengaluru, India. He obtained Bachelor Degree in Computer Science and Engineering from Bharathidasan University in 1989. He obtained his M.Tech from National Institute of Technology, Tiruchirappalli in 2006 and Ph.D. in Computer Science \& Engineering from

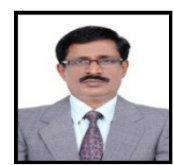
Anna University Chennai in 2014. He has presented 3 papers in International Conferences and published 17 papers in International Journals.

Mr.Ranjeet Kumar is working as an Associate Professor in the Department of Computer Science \& Engineering at Don Bosco Institute of Technology, Bengaluru 560074. He has completed Bachelor of engineering in electrical \& electronics engineering from Kuvempu University, Shimoga, Karnataka in 2001. He has also completed his Master of Technology in Computer Science \& Engineering from

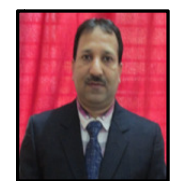
Visveswaraya Technological University, Belgaum, Karnataka in 2010. 